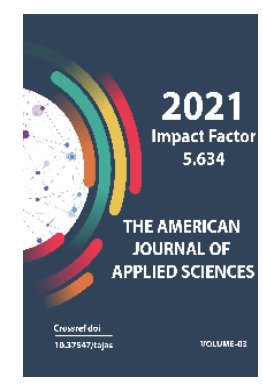

Journal Website: http://theamericanjour nals.com/index.php/taj as

Copyright: Original content from this work may be used under the terms of the creative commons attributes 4.0 licence.

\section{About One Theorem Of 2x2 Jordan Blocks Matrix}

Hojiyev Dilmurodjon Bahodirovich

Math Teacher Of The Lyceum Of Andijan State University, Uzbekistan

Muhammadjonov Akbarshoh Akramjon Og`Li

The Third-Year Student Of Andijan State University, Uzbekistan

Muzaffarova Dilshoda Botirjon Qizi

The First-Year Student Of Andijan State University, Uzbekistan

Ibrohimjonov Islombek Ilhomjon O'G' Li

The First-Year Student Of Andijan State University, Uzbekistan

Ahmadjonova Musharrafxon Dilmurod Qizi

The First-Year Student Of Andijan State University, Uzbekistan

\title{
ABSTRACT
}

In this paper, we have studied one theorem on $2 \times 2$ Jordan blocks matrix. There are 4 important statements which is used for proving other theorems such as in the differensial equations. In proving these statements, we have used mathematic induction, norm of matrix, Taylor series of $f(x)=e^{X}$.

\section{KEYWORDS}

Matrix, vector, mathematic induction method, equality, inequality, norm, expansion of series, estimation.

\section{INTRODUCTION}

First of all, we present the Theorem that is the main result of our article, then we prove statements one after another in the Theorem.

Theorem. If

$A_{i}=\left[\begin{array}{cc}-\lambda_{i} & 1 \\ 0 & -\lambda_{i}\end{array}\right], \quad \lambda_{i} \geq 0 \quad i=1,2, \ldots \quad t \in[0 ; T]$

then the following statements are true: 

1) $\mathrm{e}^{\mathrm{A}_{\mathrm{i}} \mathrm{t}}=\mathrm{e}^{-\lambda_{\mathrm{i}} \mathrm{t}}\left[\begin{array}{ll}1 & \mathrm{t} \\ 0 & 1\end{array}\right], \quad \mathrm{i}=1,2, \ldots$
2) $e^{A_{i}(t+h)}=e^{A_{i} t} \cdot e^{A_{i} h}, h \in R$.
3) $\left\|\mathrm{e}^{\mathrm{A}_{\mathrm{i}} \mathrm{t}}\right\| \leq 1+\mathrm{T}$
4) $\left\|\mathrm{e}^{\mathrm{A}_{\mathrm{i}} \mathrm{t}}-\mathrm{E}_{2}\right\| \leq \mathrm{t}, \quad \mathrm{E}_{2}=\left[\begin{array}{ll}1 & 0 \\ 0 & 1\end{array}\right]$,

where $T$ is sufficiently large fixed number, $\|A\|=\max _{|x|=1}|A x|$.

Proof. We would prefer to present proofs in order which is shown above, respectively.

1. Here, the following Taylor expansion of $\mathrm{f}(\mathrm{x})=\mathrm{e}^{\mathrm{x}}$ function is important to prove 1) equality, so we use this

$$
e^{x}=1+x+\frac{x^{2}}{2 !}+\frac{x^{3}}{3 !}+\ldots+\frac{x^{n}}{n !}+\ldots=\sum_{n=0}^{\infty} \frac{x^{n}}{n !}
$$

If we mean $\mathrm{x}=\mathrm{A}_{\mathrm{i}} \mathrm{t}$, then we get a result that:

$$
e^{A_{i} t}=E_{2}+A_{i} t+\frac{\left(A_{i} t\right)^{2}}{2 !}+\frac{\left(A_{i} t\right)^{3}}{3 !}+\ldots+\frac{\left(A_{i} t\right)^{n}}{n !}+\ldots=\sum_{n=0}^{\infty} \frac{\left(A_{i} t\right)^{n}}{n !}
$$

We establish one by one $A_{i}^{n}(n=2,3, \ldots)$ in (2) equality for convenience:

$$
\begin{aligned}
A_{i}{ }^{2} & =\left[\begin{array}{cc}
-\lambda_{\mathrm{i}} & 1 \\
0 & -\lambda_{\mathrm{i}}
\end{array}\right] \cdot\left[\begin{array}{cc}
-\lambda_{\mathrm{i}} & 1 \\
0 & -\lambda_{\mathrm{i}}
\end{array}\right]=\left[\begin{array}{cc}
\lambda_{\mathrm{i}}{ }^{2} & -2 \lambda_{\mathrm{i}} \\
0 & \lambda_{\mathrm{i}}{ }^{2}
\end{array}\right] \\
\mathrm{A}_{\mathrm{i}}{ }^{3} & =\left[\begin{array}{cc}
\lambda_{\mathrm{i}}{ }^{2} & -2 \lambda_{\mathrm{i}} \\
0 & \lambda_{\mathrm{i}}{ }^{2}
\end{array}\right] \cdot\left[\begin{array}{cc}
-\lambda_{\mathrm{i}} & 1 \\
0 & -\lambda_{\mathrm{i}}
\end{array}\right]=\left[\begin{array}{cc}
-\lambda_{\mathrm{i}}{ }^{3} & 3 \lambda_{\mathrm{i}}{ }^{2} \\
0 & -\lambda_{\mathrm{i}}{ }^{3}
\end{array}\right] \\
\mathrm{A}_{\mathrm{i}}{ }^{4} & =\left[\begin{array}{cc}
-\lambda_{\mathrm{i}}{ }^{3} & 3 \lambda_{\mathrm{i}}{ }^{2} \\
0 & -\lambda_{\mathrm{i}}{ }^{3}
\end{array}\right] \cdot\left[\begin{array}{cc}
-\lambda_{\mathrm{i}} & 1 \\
0 & -\lambda_{\mathrm{i}}
\end{array}\right]=\left[\begin{array}{cc}
\lambda_{\mathrm{i}}{ }^{4} & -4 \lambda_{\mathrm{i}}{ }^{3} \\
0 & \lambda_{\mathrm{i}}{ }^{4}
\end{array}\right]
\end{aligned}
$$

As a result of continue doing this process, we obtain:

$$
A_{i}{ }^{n}=\left[\begin{array}{cc}
(-1)^{\mathrm{n}} \lambda_{i}{ }^{n} & (-1)^{\mathrm{n}-1} \cdot \mathrm{n} \cdot \lambda_{\mathrm{i}}{ }^{\mathrm{n}-1} \\
0 & (-1)^{\mathrm{n}} \cdot \lambda_{\mathrm{i}}{ }^{\mathrm{n}}
\end{array}\right]
$$


Now, we indicate that (3) equality which we mentioned for $\mathrm{A}_{\mathrm{i}}{ }^{\mathrm{n}}$ by inductive method is correct for any $\mathrm{n} \in \mathrm{N}$ by using mathematic induction method so we prove that

$$
\begin{gathered}
A_{i}{ }^{n+1}=\left[\begin{array}{cc}
(-1)^{n+1} \lambda_{i}{ }^{n+1} & (-1)^{n} \cdot(n+1) \cdot \lambda_{i}{ }^{n} \\
0 & (-1)^{n+1} \cdot \lambda_{i}{ }^{n+1}
\end{array}\right] \\
A_{i}^{n+1}=A_{i}^{n} \cdot A_{i}= \\
=\left[\begin{array}{cr}
\left(-\lambda_{i}\right)^{n} & n \cdot\left(-\lambda_{i}\right)^{n-1} \\
0 & \left(-\lambda_{i}\right)^{n}
\end{array}\right] \cdot\left[\begin{array}{cc}
-\lambda_{i} & 1 \\
0 & -\lambda_{i}
\end{array}\right]= \\
=\left[\begin{array}{cr}
(-1)^{n+1} \lambda_{i}{ }^{n+1} & (-1)^{n} \cdot(n+1) \cdot \lambda_{i}{ }^{n} \\
0 & (-1)^{n+1} \cdot \lambda_{i}{ }^{n+1}
\end{array}\right]
\end{gathered}
$$

This indicates that (3) equality is correct for any $\mathrm{n} \in \mathrm{N}$.

Therefore,

$$
\begin{gathered}
e^{A_{i} t}=\sum_{n=0}^{\infty} \frac{\left(A_{i} \cdot t\right)^{n}}{n !}=\sum_{n=0}^{\infty} \frac{A_{i}^{n} \cdot t^{n}}{n !}= \\
\sum_{n=0}^{\infty}\left[\begin{array}{cc}
\frac{\left(-\lambda_{i} t\right)^{n}}{n !} & \frac{\left(-\lambda_{i} t\right)^{n-1}}{(n-1) !} \cdot t \\
0 & \frac{\left(-\lambda_{i} t\right)^{n}}{n !}
\end{array}\right]=e^{-\lambda_{i}} \cdot\left[\begin{array}{ll}
1 & t \\
0 & 1
\end{array}\right]
\end{gathered}
$$

1. Next, we indicate that 2) equality is right.

If we mean $x=A_{i}(t+h)$ and close to the growth of (1) we can write the left hand of 2) equality, we get a result

$$
\begin{gathered}
e^{A_{i}(t+h)}=E_{3}+A_{i}(t+h)+\frac{\left[A_{i}(t+h)\right]^{2}}{2 !}+\frac{\left[A_{i}(t+h)\right]^{3}}{3 !}+\ldots \\
+\ldots+\frac{\left[A_{i}(t+h)\right]^{n}}{n !}+\ldots+=E_{2}+A_{i}(t+h)+A_{i}^{2}\left(\frac{t^{2}}{2 !}+t \cdot h+\frac{h^{2}}{2 !}\right)+\ldots
\end{gathered}
$$




$$
\begin{gathered}
+\ldots+A_{i}^{n}\left[\frac{t^{n}}{n !}+\frac{t^{n-1}}{n-1} \cdot \frac{h}{1 !}+\ldots+\frac{t^{n-k}}{(n-k) !} \cdot \frac{h^{k}}{k !}+\ldots+\frac{h^{n}}{n !}\right]+\ldots= \\
=\left(E_{2}+A_{i} t+\frac{\left(A_{i} t\right)^{2}}{2 !}+\ldots+\frac{\left(A_{i} t\right)^{n}}{n !}+\ldots\right)\left(E_{2}+A_{i} h+\frac{\left(A_{i} h\right)^{2}}{2 !}+\ldots+\frac{\left(A_{i} h\right)^{n}}{n !}+\ldots\right) \\
=e^{A_{i} t} \cdot e^{A_{i} h}
\end{gathered}
$$

Proof is completed.

2. Next, we prove 3) inequality

In fact, we get a result from 1) that

$$
\left\|\mathrm{e}^{\mathrm{A}_{\mathrm{i}} \mathrm{t}}\right\|=\left\|\mathrm{e}^{-\lambda_{\mathrm{i}}} \cdot\left[\begin{array}{ll}
1 & \mathrm{t} \\
0 & 1
\end{array}\right]\right\| \leq\left\|\left[\begin{array}{ll}
1 & \mathrm{t} \\
0 & 1
\end{array}\right]\right\|
$$

According to the original estimate $\|\mathrm{A}\|=\max _{|\mathrm{x}|=1}|\mathrm{Ax}|$ above:

$$
\begin{gathered}
\left\|\mathrm{e}^{\mathrm{A}_{\mathrm{i}} \mathrm{t}}\right\| \leq\left\|\left[\begin{array}{ll}
1 & \mathrm{t} \\
0 & 1
\end{array}\right]\right\|=\max _{|\mathrm{x}|=1}\left|\left[\begin{array}{ll}
1 & \mathrm{t} \\
0 & 1
\end{array}\right]\left[\begin{array}{l}
\mathrm{x}_{1} \\
\mathrm{x}_{2}
\end{array}\right]\right|= \\
\max _{|\mathrm{x}|=1}\left|\left[\begin{array}{c}
\mathrm{x}_{1}+\mathrm{t} \cdot \mathrm{x}_{2} \\
\mathrm{x}_{2}
\end{array}\right]\right|
\end{gathered}
$$

where $x=\left[\begin{array}{l}x_{1} \\ x_{2}\end{array}\right],|x|=\sqrt{x_{1}^{2}+x_{2}^{2}}=1$

We mean $a, b, c$ vectors by

$$
\begin{gathered}
\mathrm{a}=\left[\begin{array}{l}
\mathrm{x}_{1} \\
\mathrm{x}_{2}
\end{array}\right], \mathrm{b}=\left[\begin{array}{c}
\mathrm{t} \cdot \mathrm{x}_{2} \\
0
\end{array}\right], \mathrm{c}=\left[\begin{array}{l}
0 \\
0
\end{array}\right] \\
{\left[\begin{array}{c}
\mathrm{x}_{1}+\mathrm{t} \cdot \mathrm{x}_{2} \\
\mathrm{x}_{2}
\end{array}\right]=\left[\begin{array}{l}
\mathrm{x}_{1} \\
\mathrm{x}_{2}
\end{array}\right]+\left[\begin{array}{c}
\mathrm{t} \cdot \mathrm{x}_{2} \\
0
\end{array}\right]+\left[\begin{array}{l}
0 \\
0
\end{array}\right]=\mathrm{a}+\mathrm{b}+\mathrm{c}} \\
\left\|\mathrm{e}^{\mathrm{A}_{\mathrm{i}} \mathrm{t}}\right\| \leq \max _{|\mathrm{x}|=1}|\mathrm{a}+\mathrm{b}+\mathrm{c}|
\end{gathered}
$$

We know that $|a+b+c| \leq|a|+|b|+|c|$ inequality is correct for any $a, b, c$ vectors. By using this, 


$$
\begin{aligned}
\left\|\mathrm{e}^{\mathrm{A}_{\mathrm{i}} \mathrm{t}}\right\| & \leq \max _{|\mathrm{x}|=1}|\mathrm{a}+\mathrm{b}+\mathrm{c}| \leq|\mathrm{a}|+|\mathrm{b}|+|\mathrm{c}|= \\
& =\sqrt{\mathrm{x}_{1}^{2}+\mathrm{x}_{2}^{2}}+\sqrt{\mathrm{t}^{2} \cdot \mathrm{x}_{2}^{2}}= \\
& =1+\mathrm{t} \cdot{\sqrt{\mathrm{x}_{2}^{2}}}^{2} \leq 1+\mathrm{t}
\end{aligned}
$$

because of $t \in[0 ; T]$, we get a result

$$
\left\|\mathrm{e}^{\mathrm{A}_{\mathrm{i}} \mathrm{t}}\right\| \leq 1+\mathrm{T}
$$

3. Eventually, we move to prove 4) inequality in the Theorem:

$$
\left\|\mathrm{e}^{\mathrm{A}_{\mathrm{i}} \mathrm{t}}-\mathrm{E}_{2}\right\| \leq \mathrm{t}
$$

where $\mathrm{E}_{2}=\left[\begin{array}{ll}1 & 0 \\ 0 & 1\end{array}\right]$.

We have the following expressions by using 1 ) equality and $\mathrm{e}^{-\lambda_{\mathrm{i}} \mathrm{t}} \leq 1$

inequality:

$$
\begin{gathered}
\left\|\mathrm{e}^{\mathrm{A}_{\mathrm{i}} \mathrm{t}}-\mathrm{E}_{2}\right\|=\left\|\mathrm{e}^{-\lambda_{\mathrm{i}} \mathrm{t}}\left[\begin{array}{ll}
1 & \mathrm{t} \\
0 & 1
\end{array}\right]-\left[\begin{array}{ll}
1 & 0 \\
0 & 1
\end{array}\right]\right\| \leq \\
\leq\left\|\left[\begin{array}{ll}
1 & \mathrm{t} \\
0 & 1
\end{array}\right]-\left[\begin{array}{ll}
1 & 0 \\
0 & 1
\end{array}\right]\right\|=\left\|\begin{array}{ll}
0 & \mathrm{t} \\
0 & 0
\end{array}\right\|= \\
\quad=\max _{|\mathrm{x}|=1}\left|\left[\begin{array}{ll}
0 & \mathrm{t} \\
0 & 0
\end{array}\right]\left[\begin{array}{c}
\mathrm{x}_{1} \\
\mathrm{x}_{2}
\end{array}\right]\left\|=\max _{|\mathrm{x}|=1} \mid\left[\begin{array}{c}
\mathrm{t} \cdot \mathrm{x}_{2} \\
0
\end{array}\right]\right\|\right.
\end{gathered}
$$

where $x=\left[\begin{array}{l}x_{1} \\ x_{2}\end{array}\right],|x|=\sqrt{x_{1}^{2}+x_{2}^{2}}=1$.

Close to the proof of the third property we mean a, b, c vectors by

$$
\mathrm{a}=\left[\begin{array}{c}
\mathrm{t} \cdot \mathrm{x}_{2} \\
0
\end{array}\right], \mathrm{b}=\left[\begin{array}{l}
0 \\
0
\end{array}\right], \mathrm{c}=\left[\begin{array}{l}
0 \\
0
\end{array}\right]
$$

And according to $|\mathrm{a}+\mathrm{b}+\mathrm{c}| \leq|\mathrm{a}|+|\mathrm{b}|+|\mathrm{c}|$ inequality 


$$
\begin{gathered}
\left\|\mathrm{e}^{\mathrm{A}_{\mathrm{i}} \mathrm{t}}-\mathrm{E}_{2}\right\| \leq \max _{|\mathrm{x}|=1}|\mathrm{a}+\mathrm{b}+\mathrm{c}| \leq[|\mathrm{a}|+|\mathrm{b}|+|\mathrm{c}|]_{|\mathrm{x}|=1}= \\
\sqrt{\mathrm{t}^{2} \cdot \mathrm{x}_{2}^{2}}=\mathrm{t} \cdot \sqrt{\mathrm{x}_{2}^{2}} \leq \mathrm{t} .
\end{gathered}
$$

This Theorem is proved completely.

\section{REFERENCES}

1. V.G. Miladjanov, R.V. Mullajonov, K.X. Turg'unova, SH.N. Abdug'apporova, J.V. Mullajonova, "Matritsalar nazariyasining tanlangan boblari”, Toshkent 2014.

2. Р.В.Белман ведение теории матриц.-М., Наука, 1976.

3. А. П. Гроссман, Р. Фрезер, В. Дункан и А. Коллар, “Теория матриц и её приложения к дифференциальным уравнениям и динамике”, УМН, 1952, том 7, выпуск 3(49), 190192

4. Bhatia, R. (1997). Matrix Analysis. Graduate Texts in Mathematics. 169. Springer. ISBN 978-0-387-94846-1.

5. Householder, Alston S. (2006). The Theory of Matrices in Numerical Analysis. Dover Books on Mathematics. ISBN 978-0-486-44972-2.

6. Van Kortryk, T. S. (2016). "Matrix exponentials, SU(N) group elements, and real polynomial roots". Journal of Mathematical Physics. 57 (2): 021701. arXiv:1508.05859. 